\title{
ORDERS IN SEMILOCAL RINGS ${ }^{1}$
}

\author{
BY CARL FAITH
}

Communicated by H. Bass, April 16, 1971

A semilocal ring $S$ is one such that $S / \operatorname{rad} S$ is semisimple (= a direct sum of simple modules). The first theorem generalizes a theorem of Faith and Utumi [65] to semilocal rings, which had been extended by Robson [67] to artinian rings. (Cf. also Procesi [65].)

(A) THEOREM. If $R$ has a semilocal right quoring (=quotient ring) $S=D_{n}$ which is a full $n \times n$ matrix ring, then there exists a set $M$ of $n \times n$ matrix units such that $R$ contains a right order $F_{n}$ of $D_{n}$, and $F$ is a right order in $D=$ centralizer $M$.

The proof depends on the lemma.

(B) Leмma. If $F_{n}$ has semilocal right quoring $D_{n}$, where $F$ is subring of $D$, then for every regular element $t \in F_{n}$, there exists a regular element $a \in F_{n}$ such that $x=t a=\left(x_{i j}\right)$ is represented by a matrix $\left(x_{i j}\right)$ with diagonal elements $x_{i i}$ regular elements of $F$, and the off-diagonal elements $x_{i j}$ are contained in $F \cap \mathrm{rad} D$.

This was proved by Faith-Utumi [65] for semisimple $D$, and Robson [67] for artinian $D$. The proof of (A) makes use only of the case $D$ is semisimple, and both (B) and (A) require the rather obvious fact that if $R$ has semilocal right quoring $S$, then $\bar{R}$ has a semisimple right quoring $\bar{S}=S / \operatorname{rad} S$, where $\bar{R} \approx R /(R \cap \operatorname{rad} S)$ is the image of $R$ under the canonical map $S \rightarrow \bar{S}$. Then, $\bar{R}$ is semiprime right Goldie.

(A) has the following application.

(C) THEOREM. Any maximal $\sim_{l}$ right order in a semilocal ring $S=D_{n}$ is isomorphic to the endomorphism ring of a torsion-free unital module over a right order of $D$.

The proof is patterned after that of Faith [64] for the case $D$ is a field, and Robson [67] for $D$ artinian. (Note. Two right orders $R_{1}$ and

AMS 1970 subject classifications. Primary 16A08, 16A46, 16A52; Secondary 16A42.

Key words and phrases. Artinian, closed right ideal, matrix ring, noetherian, nilpotent ideal, right order, quotient ring, quorite, perfect ring, regular element, reflective ideal, semilocal ring, semiprime ring, semisimple ring, semiprimary ring, torsionfree module, right vanishing radical.

1 The research in this paper was supported in part by a grant from the National Science Foundation. 
$R_{2}$ are left equivalent, notation $R_{1} \sim_{l} R_{2}$, provided that $R_{1} b \subseteq R_{2}$ and $R_{2} a \subseteq R_{1}$ for two regular elements $a$ and $b$ of $S$.)

Any right self-injective semilocal ring $D$ is a quotient ring, a fact that shows that (A) holds for rings more general than $D$ artinian. Thus, (A) holds for a ring which is right $P F$ in the sense of Azumaya [66] (cf. Kato [68], Onodera [68], Osofsky [66], and Utumi [67]). An ideal $T$ is reflective if and only if

$$
c \in R \text { is regular } \Leftrightarrow[c+T] \text { is regular in } R / T \text {. }
$$

A right ideal $I$ is a $q$-regular right ideal if $c+x$ is regular in $R$ for all regular $c \in R$, and all $x \in I$. Every reflective ideal is $q$-regular. An ideal $T$ is right quorite provided that for every regular $c \in R$, and every $x \in T$, there is a regular $c_{1} \in R$, and an element $x_{1} \in T$ such that $x c_{1}=c x_{1}$. (Mnemonic. $c^{-1} x=x_{1} c_{1}^{-1}$.) If $R$ has a right quoring $S$, then every closed ideal of $R$ is right quorite. An ideal $T$ is (semiprime) right Goldie provided that $R / T$ is (semiprime) right Goldie.

(D) THEOREM. A ring $R$ has a semilocal right quoring if and only if the sum $T$ of all the q-regular right ideals of $R$ is a reflective, right quorite, and right semiprime Goldie ideal.

The proof depends upon the theorem of Feller and Swokowski [61] which asserts that $R$ has a right quoring whenever $R$ has a reflective, right quorite $S$ ideal $T$ such that $R / T$ has a right quoring $Q$, and in this case $T S=S T S$, and $T S \cap R=T$. Furthermore, it is clear that every reflective ideal is $q$-regular. Therefore, the condition of Theorem (D) is the assertion that there exists a maximal q-regular ideal, reflective, quorite ideal $T$ which contains every $q$-regular right ideal. This is reminiscent of (and indeed, $q$-regularity generalizes) the Perlis-Jacobson characterization of the radical of a ring.

(E) Corollary. A ring $R$ has a semilocal right quoring $S$ which is: (a) right noetherian, (b) right artinian, (c) a ring with right vanishing radical, (d) semiprimary if and only if $R$ contains an ideal $T$ with the properties stated in (D) and with the respective properties;

(a) $R$ satisfies the a.c.c. on closed right ideals.

(b) $T$ is nilpotent, and $R$ satisfies the a.c.c. on closed right ideals. (Then $R$ satisfies the d.c.c. on closed right ideals, and conversely.)

(c) $T$ is right vanishing (=right $T$-nilpotent).

(d) $T$ is nilpotent.

In the case (c), then $R$ is left perfect by Bass's theorem [60], so this characterizes rings with perfect right quotient rings. Cf. Jategaonkar [69], Gupta [68], Gupta and Saha [67], Robson [67], 
Small [66], and Talintyre [66]. For other pertinent references to classical quotient rings, including those which are quasi-Frobenius, consult Elizarov [69].

\section{REFERENCES}

66. G. Azumaya, Completely faithful modules and self-injective rings, Nagoya Math. J. 27 (1966), 697-708. MR 35 \#4253.

60. H. Bass, Finitistic dimension and a homological generalization of semi-primary rings, Trans. Amer. Math. Soc. 95 (1960), 466-488. MR 28 \#1212.

69. V. P. Elizarov, Quotient rings, Algebra and Logic 8 (1969), 219-243.

64. C. Faith, Noetherian simple rings, Bull. Amer. Math. Soc. 70 (1964), 730-731. MR 29 \#4773.

65. C. Faith and Y. Utumi, On Noetherian prime rings, Trans. Amer. Math. Soc. 114 (1965), 53-60. MR $30 \# 3111$.

61. E. H. Feller and E. W. Swokowski, Reflective $N$-prime rings with the ascending chain condition, Trans. Amer. Math. Soc. 99 (1961), 264-271; erratum, ibid. 101 (1961), 555. MR $22 \# 11011 ; 22 \# 2547$.

61. - Reflective rings with the ascending chain condition, Proc. Amer. Math. Soc. 12 (1961), 651-653. MR 23 \#A3158.

58. A. W. Goldie, The structure of prime rings under ascending chain conditions, Proc. London Math. Soc. (3) 8 (1958), 589-608. MR 21 \#1988.

60. - Semi-prime rings with maximum condition, Proc. London Math. Soc.

(3) 10 (1960), 201-220. MR $22 \# 2627$.

67. R. N. Gupta and F. Saha, Remarks on a paper of Small, J. Math. Sci. 2 (1967), 7-16. MR 36 \#2642.

68. R. N. Gupta, Characterisation of rings whose classical quotient rings are perfect rings, Osaka J. Math. 5 (1968), 69-87.

45. N. Jacobson, The radical and semi-simplicity for arbitrary rings, Amer. J. Math. 67 (1945), 300-320. MR 7, 2.

69. A. V. Jategaonkar, Orders in Artinian rings, Bull. Amer. Math. Soc. 75 (1969), 1258-1259. MR $40 \# 176$.

68. T. Kato, Some generalization of QF-rings, Proc. Japan Acad. 44 (1968), 114119. MR 38* \#183.

68. T. Onodera, Über Kogeneratoren, Arch. Math. (Basel) 19 (1968), 402-410. MR 38 \#2170.

66. B. L. Osofsky, A generalization of quasi-Frobenius rings, J. Algebra 4 (1966), 373-387. MR 34 \#4305.

42. S. Perlis, $A$ characterization of the radical of an algebra, Bull. Amer. Math. Soc. 48 (1942), 128-132. MR 3, 264.

65. C. Procesi, Su un teorema di Faith-Utumi, Rend. Mat. e Appl. (5) 24 (1965), 346-347. MR 34 \#4302.

67. J. C. Robson, Artinian quotient rings, Proc. London Math. Soc. (3) 17 (1967), 600-616. MR $36 \# 199$.

66. L. Small, Orders in Artinian rings, J. Algebra 4 (1966), 13-41. MR 34 \#199.

66. T. D. Talintyre, Quotient rings with minimum condition on right ideals, J. London Math. Soc. 41 (1966), 141-144. MR 36 \#6445.

67. Y. Utumi, Self-injective rings, J. Algebra 6 (1967), 56-64. MR 35 \#219.

Rutgers University, New Brunswick, New Jersey 08903 